\title{
Tissue transglutaminase as a central mediator in inflammation-induced progression of breast cancer
}

\author{
Navneet Agnihotri, ${ }^{1,2}$, Santosh Kumar ${ }^{1,3}$ and Kapil Mehta*1
}

\begin{abstract}
TGM2 is a stress-responsive gene that encodes a multifunctional and structurally complex protein called tissue transglutaminase (abbreviated as TG2 or tTG). TGM2 expression is frequently upregulated during inflammation and wounding. Emerging evidence indicates that TGM2 expression is aberrantly upregulated in multiple cancer cell types, particularly those selected for resistance to chemotherapy and radiation therapy and those isolated from metastatic sites. It is becoming increasingly evident that chronic expression of TG2 in epithelial cancer cells initiates a complex series of signaling networks which contributes to the development of drug resistance and an invasive phenotype. For example, forced or basal high expression of TG2 in mammary epithelial cells is associated with activation of nuclear transcription factor-kappa B (NF-kB), Akt, focal adhesion kinase, and hypoxia-inducible factor. All of these changes are considered hallmarks of aggressive tumors. TG2 expression is able to induce the developmentally regulated program of epithelial-to-mesenchymal transition (EMT) and to confer cancer stem cell (CSC) traits in mammary epithelial cells; both EMT and CSCs have been implicated in cancer metastasis and resistance to standard therapies. Importantly, TG2 expression in tumor samples is associated with poor disease outcome, increased drug resistance, and increased incidence of metastasis. These observations imply that TG2 plays a crucial role in promoting an aggressive phenotype in mammary epithelial cells. In this review, we discuss recent evidence that TG2regulated pathways contribute to the aggressive phenotype in breast cancer.
\end{abstract}

*Correspondence: kmehta@mdanderson.org

'Department of Experimental Therapeutics, Unit 1950, The University of Texas M. D. Anderson Cancer Center, 1515 Holcombe Boulevard, Houston, TX 77030, USA Full list of author information is available at the end of the article

\section{Introduction}

Breast cancer is the most common malignancy diagnosed among women worldwide and is the second leading cause of cancer-related deaths in women [1]. Early detection, improved surgical techniques, and targeted therapies have resulted in a general downward trend in the prevalence of the disease. However, recurrence of cancer owing to metastasis and emergence of drug resistance still account for more than $90 \%$ of cancer-related deaths and continue to pose major clinical challenges in the successful treatment of the disease. The high rate of relapse in patients with breast cancer - estimated to be approximately $30 \%$ - underscores the need to identify tumorencoded genes and to understand how these genes contribute to metastasis. Such an understanding will enable novel strategies (a) to prevent the progression of early lesions to metastatic disease, (b) to treat metastatic disease, and (c) to stratify tumors with high metastatic potential.

Although genetic changes are central to many aspects of cancer development, they are not sufficient to cause disease progression. In addition to these genetic alterations, early-stage tumors (for example, ductal carcinoma in situ (DCIS) of the breast) require some ancillary changes (induced by the tumor microenvironment) to become invasive and to metastasize [2]. Many inflammatory mediators produced in the tumor milieu can induce persistent epigenetic changes that affect fundamental processes necessary for generating tumor cell variants with metastatic ability [2]. However, information on how and what inflammation-induced epigenetic changes affect invasion and metastasis remains elusive.

Recently, we identified a novel inflammatory pathway that is constitutively activated in cancer cells and promotes drug resistance and an invasive phenotype in epithelial cancer cells [3]. Central to this pathway is increased expression of the structurally and functionally complex protein called tissue transglutaminase (TG2 or tTG). In this review, we discuss the evidence that aberrant expression of TG2 promotes a metastatic and drug-resistant phenotype in breast epithelial cells by inducing the developmentally regulated program of epithelial-to- mesenchymal transition (EMT) and conferring stem cell traits to the cells. 


\section{TG2: the multifunctional protein}

TG2 is the most complex and ubiquitous member of the transglutaminase family of enzymes that catalyze posttranslational modification of proteins by introducing highly stable $\varepsilon$ - $(\gamma$-glutamyl)lysine isopeptide linkages or by incorporating polyamines at selected peptide-bound glutamine residues in the presence of $\mathrm{Ca}^{2+}$ [4]. Besides catalyzing the protein crosslinking reactions, TG2 can hydrolyze nucleotide guanine triphosphate (GTP) and serve as a protein disulfide isomerase, as a kinase, and as a scaffold protein $[4,5]$. In the physiological context, TG2 is referred to as a component of cell or tissue defense mechanisms; its expression is frequently upregulated in response to cell damage or other stressors to protect cells from endogenous and environmental stresses and to repair tissue later for sustained homeostasis. An interesting feature of TG2 is that it can exert opposing effects depending on its localization and predominant activity.

Three important regulators of TG2 activity are $\mathrm{Ca}^{2+}$, GTP/GDP, and redox potential. Binding of $\mathrm{Ca}^{2+}$ ions to TG2 promotes its catalytically active 'open' or 'extended' conformation. Binding to GTP or GDP, in contrast, promotes the catalytically inactive 'closed' or 'compact' conformation (Figure 1). Because most intracellular TG2 is GTP-bound and calcium concentrations are low, it stays in catalytically inactive form under physiological conditions. In this compact form, TG2 serves as a scaffold protein and regulates signaling pathways by binding and altering the function or stability (or both) of certain key effector proteins. Intracellular calcium level alterations due to cell damage or other stressors induce a catalytically active (extended) conformation, resulting in TG2-catalyzed crosslinking of cellular proteins and apoptotic death (Figure 1). In contrast, TG2 in the extracellular environment, which has a considerably lower concentration of GTP/GDP and abundance of free calcium, can be expected to be present in a catalytically active form. However, most extracellular TG2 is enzymatically inactive due to disulfide bonding. Under oxidizing conditions, the inter-strand disulfide bond between Cys230 and Cys370 facilitates the formation of the more stable Cys370-Cys371 disulfide bond that inactivates TG2. The reactivation of extracellular TG2 can be achieved under favorable redox potential or alternatively by protein cofactor thioredoxin 1 . Once activated, TG2 can crosslink extracellular matrix (ECM) component proteins and stabilize the matrix for increased cell attachment, cell motility, and outside-in signaling cues [6].

\section{TG2 in breast cancer}

Several recent reports have supported increased expression of TG2 in multiple cancer types, including breast cancer [7-10]. Importantly, the increased TG2 expression is frequently associated with the development of drug resistance and a metastatic phenotype [7-10]. Aberrant expression of TG2 in breast cancer cells has been linked with epigenetic regulation of its gene (TGM2). Detailed analysis of region-specific DNA methylation revealed that TGM2 is among the selected genes whose expression is altered by hypo- and hyper-methylation status in drugresistant breast cancer cells [11]. In a later study, $\mathrm{Ai}$ and colleagues [12] observed that TGM2 gene is a promising target for epigenetic silencing in drug-resistant breast cancer cells and that its methylation status could serve as a marker for chemotherapy-sensitive breast tumors. Similarly, TG2 expression in breast tumor samples is associated with metastatic spread and poor disease outcome in patients $[8,10]$. Using loss- and gain-offunction approaches, several investigators have reported that aberrant expression of TG2 promotes resistance to chemotherapeutic drugs and invasiveness in mammary cancer cells [7-12]. TG2 is also implicated in epidermal growth factor receptor-mediated oncogenesis [13]. Activation of nuclear transcription factor-kappa B (NF$\kappa B$ ), hypoxia-inducible factor (HIF)-1 $\alpha$, Akt, and focal adhesion kinase (FAK) and downregulation of the tumor suppressor PTEN (phosphatase and tensin homologue deleted on chromosome 10) are among the common pathways that are altered in response to TG2 expression. More recently, the phosphorylation of TG2 at serine 216 was suggested to be critical for inducing NF-kB and Akt activation and downregulation of PTEN [14]. In the following section, we summarize some recent evidence that may explain the ability of TG2 to promote drug resistance and a metastatic phenotype in epithelial cancer cells.

\section{TG2-induced epithelial-to-mesenchymal transition}

It is now believed that EMT represents the first necessary step in metastatic dissemination and tumor progression. To metastasize, tumor cells must detach from the primary site, enter the circulation, and invade the distant site. All of these processes are facilitated by EMT $[15,16]$. During EMT, the polarized epithelial cells, which normally interact with the basement membrane via its basal surface, undergo multiple biochemical changes that enable them to acquire a mesenchymal phenotype. Tumor cells with the mesenchymal phenotype exhibit enhanced migratory functions and increased invasiveness and resistance to apoptosis [15]. To survive and proliferate in the distant site, tumor cells must undergo the reverse process of mesenchymal-to-epithelial transition (MET). This allows them to become established in the new microenvironment.

The occurrence of EMT in breast cancer cells is suggested by in vitro studies using breast cancer cell lines as well as in vivo by immunohistochemical studies in human invasive breast carcinomas and carcinosarcomas 


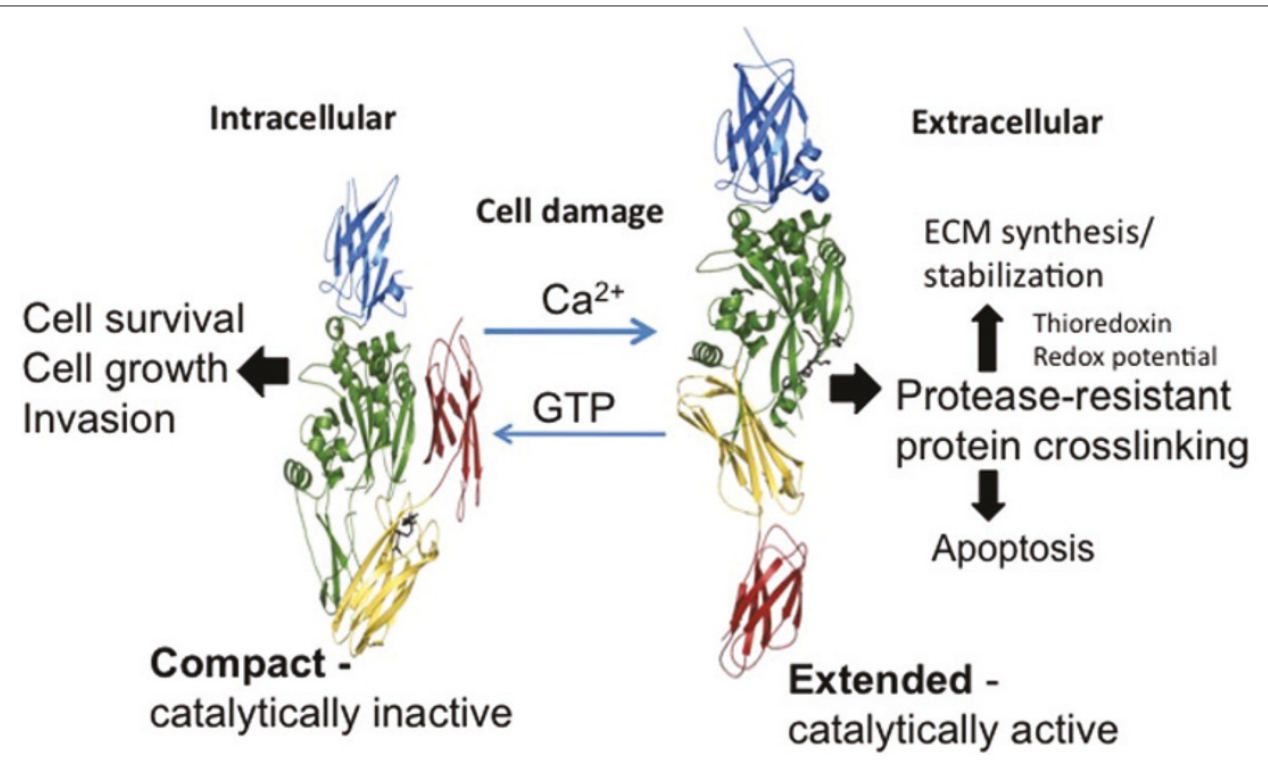

Figure 1. Allosteric regulation of tissue transglutaminase (TG2) activity and functions. TG2 is a relatively non-specific crosslinking enzyme, and its activity in and outside the cell is regulated by $\mathrm{Ca}^{2+}$, guanine nucleotides, and the redox potential. Binding of $\mathrm{Ca}^{2+}$ (dissociation constant of approximately $60 \mu \mathrm{M}$ ) is essential for TG2 to acquire a catalytically active 'open' or 'extended' conformation. In contrast, binding of GTP/GDP (dissociation constant of approximately $1.6 \mu \mathrm{M}$ ) renders TG2 in a catalytically inactive 'closed' or 'compact' conformation. Under physiological conditions, high levels of GTP, low redox potential, and low free Ca ${ }^{2+}$ level keep TG2 in its catalytically inactive compact state. However, a calcium ion influx due to extreme stress or cell damage can induce the catalytically active or'extended' conformation. In comparison with the intracellular environment, the extracellular matrix has a considerably lower GTP level and relatively high $\mathrm{Ca}^{2+}$ level. Therefore, the newly secreted TG2 can be expected to be in a catalytically active state. However, a large fraction of the extracellular TG2 in most organs is in an inactive form because of disulfide bonding. Thioredoxin 1 has been suggested to be a physiological activator of oxidized TG2. In the compact or catalytically inactive state, TG2 can act as a scaffold protein and result in the activation of various signaling pathways. In its extended and catalytically active state, TG2 catalyzes highly stable protein crosslinking, resulting in apoptotic death if inside the cell or stabilization of the matrix if outside the cell. ECM, extracellular matrix.

[17]. Further support for the argument that EMT plays a role in tumor progression comes from the observation that expression of transcription repressors Twist1 and Snail1 can be frequently detected at the DCIS stage, long before their metastatic dissemination is observed [18].

Because EMT and TG2 expression both are associated with drug resistance and the metastatic phenotype in breast cancer cells, we reasoned that the two phenomena might have some common link. Indeed, stable expression of TG2 in normal as well as in transformed mammary epithelial cells induced alterations characteristic of the EMT phenotype (Figure 2). Stable expression of TG2 in mammary epithelial cells resulted in loss of epithelial markers and gain of mesenchymal markers. Moreover, TG2-expressing cells showed a substantial increase in Snail1, Twist1, and Zeb1 transcription repressors which was accompanied by increased invasiveness and the ability to form colonies in agarose (anchorage-independent growth) [19]. Moreover, TG2 expression severely disrupted the ability of mammary epithelial cells to form organized acinar structures when grown in three-dimensional cultures [20]. Unlike TG2-deficient cells, TG2expressing cells grew into irregular spheroids with no defined lumen and fragmented basement membrane (visualized with laminin V staining). Downregulation of TG2 in drug-resistant breast cancer cells (MCF-7/Dox), which express a high basal level of TG2 and exhibit the EMT phenotype, resulted in reversal of EMT (MET), as revealed by gain in E-cadherin expression and loss of Snail1 and Zeb1 expression [19]. Moreover, tumor growth factor-beta (TGF- $\beta$ ), a potent inducer of EMT, showed complete dependence on TG2 for its ability to induce EMT. Thus, TGF- $\beta$ failed to induce EMT in mammary epithelial cells, which lacked TG2 because of stable transfection with TG2-siRNA prior to TGF- $\beta$ treatment [19]. These results suggested that TG2 is a downstream mediator of TGF- $\beta$-induced EMT. It is conceivable that TG2 represents a converging point for TGF- $\beta$-induced signaling that is critical for induction of EMT.

\section{TG2-induced epithelial-to-mesenchymal transition and stem cellness}

A growing body of evidence supports the hypothesis that cancer development is due to a small subset of cells within a tumor, termed cancer stem cells (CSCs), that uniquely initiates and sustains the disease [20]. Because CSCs exhibit intrinsic resistance to therapy, their number would be expected to increase after the treatment. 


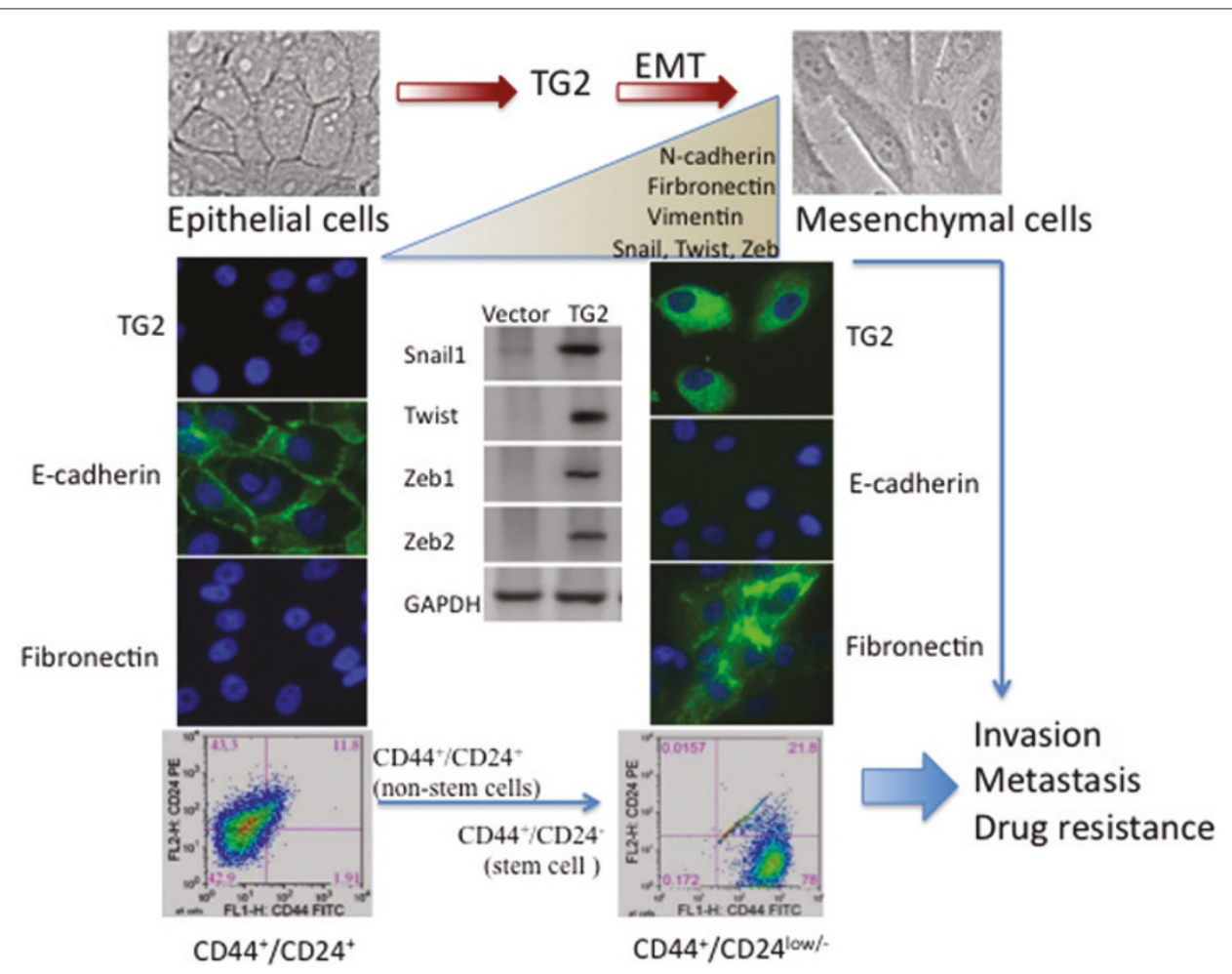

Figure 2. Tissue transglutaminase (TG2) expression induces epithelial-to-mesenchymal transition (EMT) and stem cellness in mammary epithelial cells. Stable expression of TG2 in mammary epithelial cells (shown are MCF-10A cells) resulted in appreciable morphologic and biochemical changes. Considered the hallmarks of the mesenchymal phenotype, these TG2-induced changes are associated with increased invasiveness, resistance to chemotherapeutic drugs, and acquisition of stem cell traits (increase in CD44 $/ C D 24^{\text {low/- }}$ subpopulation, increased capability to form mammospheres and self-renewal ability, and cellular plasticity).

Indeed, prolonged in vitro treatment of MCF-7 cells with doxorubicin resulted in a significant increase in a subpopulation of cells with CSC characteristics [21]. Similarly, the gene expression profile of residual tumor cells that survived chemotherapy was found to be different from that of cells in the initial tumors and closely resembled the EMT gene signature [22]. The highly tumorigenic subpopulation of breast CSCs is characterized by a CD $44^{+} / \mathrm{CD} 24^{- \text {-low }}$ antigenic phenotype. The first direct evidence that EMT and breast CSCs share some features was provided by Mani and colleagues [23], who observed that ectopic expression of Twist1 or Snail1 in immortalized human mammary epithelial cells induced EMT and augmented their ability to form mammospheres, with increased expression of CSC markers. A similar link between EMT and stem cell markers has been observed in basal-like tumors, which are known for their aggressive phenotype and high propensity to metastasize [17]. The gene signature of breast CSCs is also shared by a recently identified claudin-low subgroup of breast tumors, which display EMT-like characteristics and have high expression of vimentin and Twist [24].
In our own laboratory, we found that TG2 expression in mammary epithelial cells, in addition to altered expression of several other EMT-related genes, caused a substantial decrease in claudin 1,4 , and 8 transcript expression (S Kumar and K Mehta, unpublished observations). TG2-induced EMT was accompanied by acquisition of stem cell characteristics, as revealed by an increase in $\mathrm{CD} 44^{+} / \mathrm{CD} 24^{- \text {llow }}$ cells (Figure 2) and a concomitant decrease in $\mathrm{CD}^{-} 26^{+}$cells $[25,26]$. Moreover, TG2-expressing mammary epithelial cells showed an increased ability to form mammospheres, self-renewal ability, and plasticity. Mammospheres derived from TG2expressing MCF-10A cells differentiated into complex secondary structures when grown on Matrigel. Cells in these secondary structures differentiated into Muc1positive (luminal marker) and integrin $\alpha 6$-positive (basal marker) cells in response to prolactin treatment $[25,26]$. A similar association between TG2, EMT, and stem celllike characteristics has been reported for ovarian cancer cells [27].

These observations imply that sustained expression of TG2 induces EMT and stem cell-like traits and thus could contribute to the development of drug resistance 
and metastatic competence by breast cancer cells. Indeed, analysis of tumor samples at a 7-year follow-up of patients with breast cancer revealed that TG2 expression in primary tumors was inversely correlated with recurrence-free and metastasis-free survival [10]. Although the overall survival rates in these patients did not show significant correlation with TG2 levels, patients with elevated TG2 had shorter relapse-free survival and metastasis-free survival after adjustment for known prognostic factors such as tumor size, lymph node metastasis, age, and hormone receptor status [10]. On the basis of these observations, the authors concluded that TG2 represents an independent prognostic marker for early disease recurrence and progression to metastasis in patients with breast cancer.

\section{TG2-regulated signaling pathways}

TG2 in tumor cells serves as a double-edged sword. Both intracellular and extracellular TG2 can regulate cell signaling pathways that promote cell survival, cell motility, cell attachment, and invasive behavior (Figure 3). Previous reports have demonstrated that aberrant expression of TG2 in epithelial cells results in constitutive activation of FAK, Akt, and NF-kB [6,7]. These pathways, in turn, can contribute to the progression of cancer cells by inducing EMT and promoting drug resistance and metastasis $[15,16]$. For example, constitutively active NF- $\mathrm{kB}$ is a hallmark of many advanced-stage cancers, including breast cancer. It can upregulate multiple downstream genes that are known to protect cells from cell death, promote invasiveness, or induce EMT and stem cell properties [28]. Moreover, activation of NF-kB is considered central to inflammation-induced tumor progression. NF- $\mathrm{kB}$, in turn, regulates transcriptional regulation of TG2 via ataxia telangiectasia mutated (ATM) signaling in breast cancer cells and by binding to two independent consensus sites in the TGM2 promoter [29]. The TGM2 gene has also been proposed to serve as a chromatin target for metastatic tumor antigen (MTA1) and NF- $\mathrm{kB}$ signaling in lipopolysaccharide-stimulated cells [30]. Our recent data suggested that non-enzymatic scaffold TG2 could activate NF-kB via a novel noncanonical pathway by binding to and degradation of the inhibitory protein Iк $\mathrm{B} \alpha$ [31]. Indeed, TG2 expression has been shown to correlate inversely with the level of $\mathrm{I} k \mathrm{~B} \alpha$ in breast tumors [31,32]. Induced expression of TG2 in breast cancer cells resulted in decreased $I_{\kappa} \mathrm{B} \alpha$ level and increased NF- $\mathrm{kB}$ activity [33]. Conversely, downregulation of TG2 in drug-resistant MCF-7 cells by RNA interference resulted in increased $I_{\kappa} B \alpha$ and decreased localization of NF-kB to the nucleus [33]. How TG2 mediates $\mathrm{I} \kappa \mathrm{B} \alpha$ degradation is not yet known but is independent of the proteasomal pathway [31]. An earlier study by Kim [34] suggested that TG2-catalyzed polymerization of $I_{\kappa} \mathrm{B} \alpha$ results in its degradation and activation of NF- $\mathrm{B}$. However, our own studies demonstrated that catalytically active (wild-type) or inactive mutants (C277S and W241A) of TG2 were equally effective in mediating the degradation of $\mathrm{I} \kappa \mathrm{B} \alpha$ and inducing NF- $\mathrm{kB}$ activation in mammary epithelial cells [31]. TG2 also forms a complex with NF-kB for its recruitment to the promoter sequence of Snail, leading to transcriptional regulation of Snail [35]. Moreover, NF- $\kappa$ B-induced transcriptional regulation of HIF- $1 \alpha$ in TG2-expressing cells is also mediated by the recruitment of $\mathrm{TG} 2 / \mathrm{NF}-\mathrm{\kappa B}$ complex to the $H I F-1 \alpha$ promoter [31]. Thus, TG2-expressing mammary epithelial cells express a high basal level of HIF-1 $\alpha$ transcript and protein even under normoxic conditions. Downregulation of TG2 by gene-specific small inhibitory RNA (siRNA) attenuated NF- $k B$ activation and inhibited HIF-1 $\alpha$ expression [31].

The observation that TG2-induced activation of NF- $\mathrm{kB}$ results in high basal expression of HIF-1 $\alpha$ is of great significance and suggests that TG2 may play a central role in inflammation-regulated progression of cancer. Like TG2 expression, HIF- $1 \alpha$ expression is considered a negative prognostic factor because of its ability to promote chemoresistance, angiogenesis, invasiveness, metastasis, resistance to cell death, altered metabolism, and genomic stability [36]. Because of its instability under normoxic conditions, HIF-1 was thought to mediate the response only under hypoxic conditions. However, recent evidence supports that expression of HIF-1 protein can be upregulated even under normoxic conditions in response to certain hormones, growth factors, and cytokines [36]. In line with these observations, our data demonstrated that TG2 regulates HIF- $1 \alpha$ transcription by activating NF- $\kappa \mathrm{B}$. A recent study observed a similar effect of TG2 on HIF-1 $\alpha$ accumulation [37]. In that study, the authors concluded that TG2-catalyzed polymerization of the von Hippel-Lindau (VHL) protein results in its rapid degradation and depletion. Once again, in contrast to those authors, we used the catalytically inactive form of TG2 and observed a marked increase in both HIF-1 $\alpha$ protein and mRNA expression [31]. It is conceivable that transcriptional regulation of HIF- $1 \alpha$ by TG2 and NF- $\mathrm{kB}$ may act in concert with increased stabilization of the HIF- $1 \alpha$ protein. Moreover, TG2induced NF- $\mathrm{kB}$ and HIF- $1 \alpha$ are indispensable for induction of EMT as the expression of EMT regulators Snail, Twist, and Zeb1 transcription repressors is dependent on TG2-mediated HIF-1 $\alpha$ expression [31].

In addition to the NF- $\mathrm{B}$ and HIF-1 transcription factors, the cytokine interleukin-6 (IL-6) is an important inflammatory mediator that has been implicated in tumor initiation, metastasis, and onset of EMT. High IL6 levels in the serum are frequently associated with advanced-stage tumors, metastasis, and poor prognosis 


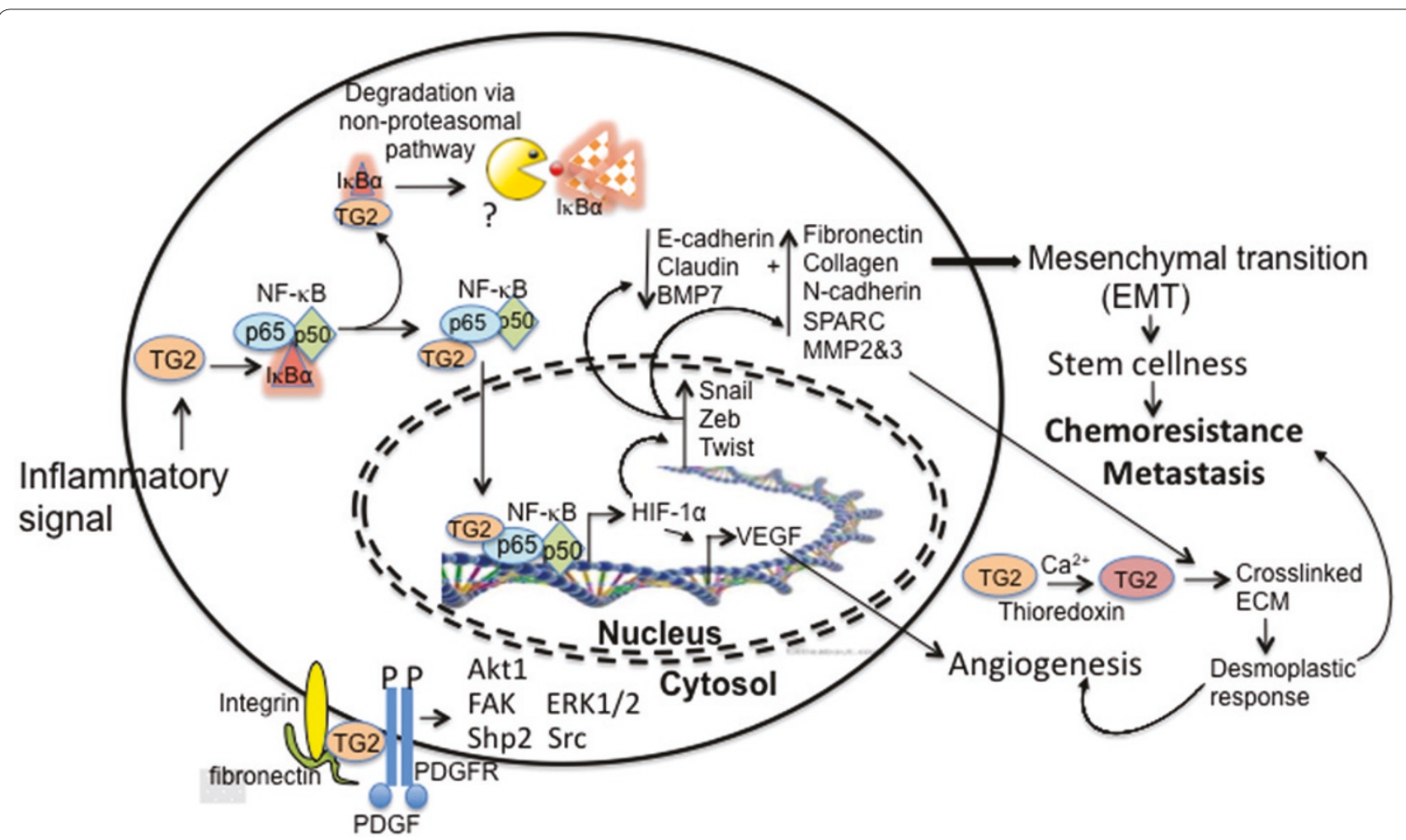

Figure 3. Tissue transglutaminase (TG2)-regulated inflammatory signaling promotes drug resistance and the metastatic phenotype. Owing to its binding and rapid degradation of the inhibitory protein $\mathrm{IKBa}_{1} \mathrm{TG} 2$ results in constitutive activation of the pro-inflammatory transcription factor NF-kB. Activated NF-kB (specifically the p65/RelA subunit), in complex with TG2, translocates to the nucleus, where it binds to HIF-1a promoter and results in its transcriptional regulation and protein expression even under normal oxygen levels. Increased expression of HIF-1a, in turn, induces the expression of transcription repressors such as Snail, Zeb, and Twist. Collectively, these TG2/NF-KB/HIF-1a-induced alterations result in acquisition of EMT and stem cell traits. Intracellular TG2 is also known to induce the synthesis and deposition of the ECM component proteins, whereas extracellular TG2 stabilizes the ECM by introducing proteolytic-resistant isopeptide bonds between its component proteins. TG2-induced changes in the ECM can alter cell attachment and cell motility functions. Similarly, TG2-catalyzed crosslinking of the ECM could further contribute to the aggressive phenotype of cancer cells by increasing shear stresses. Membrane-bound TG2, on the other hand, can interact with integrin and growth factor receptors (for example, PDGFR) to induce clustering and downstream signaling. Moreover, membranebound and integrin-associated TG2 can interact with fibronectin and further amplify integrin-mediated signaling. In a nutshell, aberrant expression of TG2 initiates reprogramming of the transcription machinery, which in turn initiates a whole new series of inside-out and outside-in signaling pathways to confer an aggressive phenotype to breast cancer cells. BMP7, bone morphogenetic protein 7; ECM, extracellular matrix; EMT, epithelialto-mesenchymal transition; ERK1/2, extracellular signal-regulated kinase 1/2; FAK, focal adhesion kinase; HIF-1, hypoxia-induced factor-1; IKBa, inhibitory KBa; MMP, matrix metalloproteinase; NF-KB, nuclear transcription factor-kappa B; PDGF, platelet-derived growth factor; PDGFR, plateletderived growth factor receptor; SPARC, secreted protein acidic and rich in cysteine; VEGF, vascular endothelial growth factor.

of breast cancer [38]. Interestingly, the production of IL-6 by cancer cells is dependent on TG2 expression, and tumor-sphere formation ability of breast cancer cells correlated with TG2-dependent IL-6 production. Both TG2 expression and downstream IL-6 production closely correlated with primary tumor growth as well as the ability of TG2-expressing MDA MB-231 cells to metastasize to the lung following their implantation in the mammary fat pads of immunocompromised mice [10]. Similarly, expression of grainyhead gene, which owing to its ability to downregulate ZEB1 has been implicated in the negative regulation of TGF- $\beta$-induced EMT [39], is downregulated in TG2-expressing MCF-10A cells, further underlining the significance of TG2 in breast cancer progression. Epidermal growth factor (EGF) is another known inducer of EMT and has also been shown to upregulate TG2 expression and to confer drug resistance phenotype in breast cancer cells [40]. It was proposed that EGF signals through Ras and c-Jun N-terminal kinase to induce TG2 expression at the leading edge of cancer cells, thus further supporting its role in breast cancer invasion and metastasis [41]. In a more recent article, Wang and colleagues [42] argued that a collaborative relationship between TG2 and EGR receptor (EGFR) is mediated by TG2 phosphorylation and suggested the use of phosphor-mutants of TG2 as a novel strategy to downregulate EGFR signaling.

In addition to orchestrating oncogenic signaling from inside the cell, the presence of TG2 in the extracellular environment (due to active cell death or passive release) 
can modulate cell growth, cell survival, and invasive signaling cues. For example, altered cell-cell (homotypic) and cell-ECM (heterotypic) interactions that are considered critical in the development and progression of cancer can be modulated by TG2. Cell surface TG2 can regulate cell adhesion and integrin-mediated signaling through direct and stable interaction with $\beta 1, \beta 3$, and $\beta 5$ integrin [6]. TG2 has strong binding affinity for the gelatin-binding region of fibronectin and thus can interact strongly with fibronectin on one hand and integrin on the other to enhance integrin (cell surface)-fibronectin (ECM) interaction. This affinity facilitates cell attachment to the matrix and also activates integrin-mediated signaling [6]. A significant impact of integrin-associated cell surface TG2 on cell adhesion, spreading, migration, survival, differentiation, ECM contraction, and ECM deposition has been observed in many transformed cells [6]. The interaction of TG2 with integrins and TG2mediated clustering of integrin potentiates outside-in signaling. For example, integrin-TG2 interaction modulates the integrin-dependent activation of FAK, Src, and p190RhoGAP, and increased levels of GTP-bound RhoA and its downstream signaling target, ROCK [6]. The net impact is the increased focal adhesion and actin stress fiber formation, leading to increased actomyosin contractility. Therefore, the interaction between integrinbound TG2 and fibronectin at the cell surface could play an important role in cancer cell metastasis and scarring.

Whereas intracellular TG2 in fibroblasts and epithelial cancer cells can accelerate the synthesis and deposition of new ECM, extracellular TG2 can stabilize it by catalyzing stable crosslinking of ECM component proteins. Therefore, it is feasible that TG2-induced changes in the ECM could contribute to a desmoplastic response in growing tumors (Figure 3). Indeed, cell lines, murine models (xenograft and genetic), and gene expression profiling studies have identified collagen, fibronectin, laminin, matrix metalloproteinases, growth factors (TGF $\beta$, plateletderived growth factor, and so on), and integrin as key constituents of the desmoplastic response. TG2 can directly or indirectly regulate the expression and function of most of these effectors. ECM remodeling and stiffening are known to affect tumor behavior. Breast cancer progression, for example, requires collagen crosslinking, ECM stiffening, and increased focal adhesion formation [43]. Lysyl oxidase-induced crosslinking of collagen results in ECM stiffening, which is associated with increased focal adhesion formation, enhanced PI3 kinase (PI3K) activity, and increased tumor invasion. The inhibition of integrin signaling repressed the invasion of premalignant epithelium into stiffened, crosslinked ECM, whereas forced integrin clustering promoted focal adhesion formation, enhanced PI3K activity, and induced invasion. On the basis of these observations, it is tempting to speculate that TG2-catalyzed crosslinking and deposition of the ECM may contribute to malignant phenotype in breast cancer.

\section{Targeting TG2}

The published evidence supports that aberrant expression of TG2 in cancer cells represents a poor prognostic marker. Its expression is associated with the transition of mammary epithelial cells into a mesenchymal state [19] and the gain of CSC traits [25]. In tumor samples, TG2 expression is associated with poor disease outcome, increased resistance to therapy, and increased incidence of metastasis. Therefore, inhibition of TG2 represents an attractive therapeutic option to reverse chemoresistance and disrupt metastatic progression. Indeed, TG2-siRNA has been successfully used for reversing chemoresistance and inhibiting metastasis in preclinical models both in vitro and in vivo $[44,45]$. However, owing to limited clinical evidence to support the effectiveness of siRNA as a therapeutic approach, its use in patients with cancer warrants further studies for optimization of siRNA delivery and safety in preclinical models.

Alternatively, small-molecule inhibitors that can bind and inhibit TG2 signaling could offer a better alternative. Increased awareness about TG2 and its role in various pathological conditions (inflammation, organ degenerative disorders, neurodegenerative disorders, celiac disease, and so on) has led to many small-molecules inhibitors that have been developed to inhibit the enzymatic activity of TG2 [46]. The most frequently used and best defined TG2 inhibitor with potential anti-cancer activity is KCC009. This inhibitor has shown some promise both in vitro and in vivo to enhance chemotherapy in various cancer models, such as ovarian, non-small lung, melanoma, breast, and colon as well as glioblastoma and meningioma [47]. However, the inference that anticancer activity of $\mathrm{KCC} 009$ is related to its ability to inhibit the enzymatic activity of TG2 needs further validation and experimentation. Many TG2 inhibitors, in addition to inhibiting its enzymatic activity by directly binding to the active site, induce conformational changes in TG2 protein [48]. For example, a structural homolog of KCC009 (ERW1227B) binds to the cysteine residue in the active side of TG2 without affecting its enzymatic activity yet was highly effective in sensitizing gliobalstoma tumors to chemotherapy [49]. These observations imply that conformational state (compact versus extended) (Figure 1) rather than catalytic activity of TG2 may be important in promoting oncogenic signaling. Indeed, our recent data using various mutant forms of TG2 supported this contention. We found that enzymatically inactive mutant forms of TG2 (C277S and W241A mutants) are fully active in inducing the EMT, CSCs, chemoresistance, invasiveness, and activation of NF-kB and HIF- $1 \alpha$ in 
mammary epithelial cells [26,31]. The GTP-binding inactive mutant (R580A), on the other hand, showed significant decrease in its ability to promote these oncogenic functions. On the basis of these observations, we propose that identification of small-molecule inhibitors that bind and lock TG2 in an oncogencially inactive state (irrespectively of their ability to inhibit enzymatic activity) can offer novel therapeutics to inhibit the progression and to reverse chemoresistance in cancer cells.

\section{Conclusions}

To summarize, we propose that sustained expression of TG2 serves as a central mediator in inflammationinduced progression of breast cancer by conferring cell survival and invasive competence on breast cancer cells (Figure 3). Specifically, TG2 expression results in constitutive activation of NF- $\mathrm{KB}$ in an IKK-independent pathway. TG2 in complex with NF-kB, in turn, binds to the $H I F-1 \alpha$ promoter and results in its transcriptional regulation and expression. Increased expression of HIF$1 \alpha$ then drives the expression of multiple downstream target genes (VEGF, glucose metabolic enzyme genes, Snail, Twist, Zeb, and so on) to promote processes such as EMT, angiogenesis, and acquisition of stem cell-like traits. This triggers altered homotypic (cell-cell) and heterotypic (cell-ECM) interactions associated with altered expression of EMT/CSC-related genes, leading to enhanced cell growth and survival in the hypoxic environment, increased synthesis of ECM proteins, and increased ability of cells to migrate and invade the surrounding tissue.

\section{Abbreviations \\ C277S, catalytic inactive mutant of tissue transglutaminase; CSC, cancer stem cell; DCIS, ductal carcinoma in situ; ECM, extracellular matrix; EGF, epidermal growth factor; EGFR, epidermal growth factor receptor; EMT, epithelial-to-mesenchymal transition; FAK, focal adhesion kinase; GTP, guanine triphosphate; HIF-1, hypoxia-induced factor-1; IL-6, interleukin-6; MET, mesenchymal-to-epithelial transition; NF-KB, nuclear transcription factor- kappa B; PI3K, PI3 kinase; PTEN, phosphatase and tensin homologue deleted on chromosome 10; siRNA, small inhibitory RNA; TG2, tissue transglutaminase; TGF- $\beta$, transforming growth factor-beta; W241A, catalytically inactive mutant of tissue transglutaminase.}

\section{Competing interests}

KM is named as an inventor on an issued patent (patent number 8,299,040, 'Methods for Treating Cancer Targeting Transglutaminase', issued Oct. 30, 2012) and a pending patent (publication number WO/2011/163198, 'TG2 Inhibitors and Uses Thereof', published Dec. 29, 2011) that are related to TG2 targeting and that are owned by the University of Texas, which employs all three authors.

\section{Authors' contributions}

$\mathrm{NA}$ and KM collected relevant references and wrote the manuscript. SK participated in discussions. All authors have read and approved the final manuscript.

\section{Acknowledgments}

This work was supported in part by the Susan G. Komen for the Cure foundation and the MD Anderson Cancer Center Support Grant (CA016672) from the National Institutes of Health. NA was supported by the Indian Council of Medical Research, India, under its International Fellowship Program. The authors wish to thank Sunita Patterson for critical reading of and editorial help with this review.

\section{Author details}

'Department of Experimental Therapeutics, Unit 1950, The University of Texas M. D. Anderson Cancer Center, 1515 Holcombe Boulevard, Houston, TX 77030, USA. '2Department of Biochemistry, Panjab University, Sector 14, Chandigarh, India 160014. 'Baylor College of Medicine, One Baylor Plaza, Houston, TX 77030, USA.

\section{Published: 25 February 2013}

\section{References}

1. Jemal A, Bray F, Center MM, Ferlay J, Ward E, Forman D: Global cancer statistics. CA Cancer J Clin 2011, 61:69-90

2. Radisky E, Radsky DC: Stromal induction of breast cancer: inflammation and invasion. Rev Endocr Metab Disord 2007, 8:279-287.

3. Mehta K, Kumar A, Kim HI: Transglutaminase 2: a multi-tasking protein in the complex circuitry of inflammation and cancer. Biochem Pharmacol 2010, 80:1921-1929.

4. Lorand L, Graham RM: Transglutaminases: crosslinking enzymes with pleiotropic functions. Nat Rev Mol Cell Biol 2003, 4:140-156.

5. Kiraly $R$, Demeny $M$, Fesus $L$ : Protein transamidation by transglutaminase 2 in cells: a disputed $\mathrm{Ca}^{2+}$-dependent action of a multifunctional protein. FEBS J 2011, 278:4717-4739.

6. Nurminskaya MV, Belkin AM: Cellular functions of tissue transglutaminase. Int Rev Cell Mol Biol 2012, 294:1-97.

7. Verma A, Mehta K: Tissue transglutaminase-mediated chemoresistance in cancer cells. Drug Resist Updat 2007, 10:144-151.

8. Mehta K, Fok J, Miller FR, Koul D, Sahin AA: Prognostic significance of tissue transglutaminase in drug resistant and metastatic breast cancer. Clin Cancer Res 2004, 10:8068-8076.

9. Mangala LS, Fok Y, Zorrilla-Calancha IR, Verma A, Mehta K: Tissue transglutaminase expression promotes cell attachment, invasion and survival in breast cancer cells. Oncogene 2007, 26:2459-2470.

10. Oh K, Ko E, Kim HS, Park AK, Moon HG, Noh DY, Lee DS: Transglutaminase 2 facilitates the distant hematogenous metastasis of breast cancer by modulating interleukin-6 in cancer cells. Breast Cancer Res 2011, 13:R96.

11. Chekhun VF, Lukyanova NY, Kovalchuk O, Tryndyak VP, Pogribny IP: Epigenetic profiling of multidrugresistant human MCF-7 breast adenocarcinoma cells reveals novel hyper and hypomethylated targets. Mol Cancer Ther 2007, 6:1089-1098.

12. Ai L, Kim WJ, Demircan B, Dyer LM, Bray KJ, Skehan RR, Massoll NA, Brown KD: The transglutaminase 2 gene (TGM2), a potential molecular marker for chemotherapeutic drug sensitivity, is epigenetically silenced in breast cancer. Carcinogenesis 2008, 29:510-518.

13. Li B, Antonyak MA, Druso JE, Cheng L, Nikitin AY, Cerione RA: EGF potentiated oncogenesis requires a tissue transglutaminase-dependent signaling pathway leading to Src activation. Proc Natl Acad SciU S A 2010, 107:1408-1413.

14. Wang $Y$, Ande SR, Mishra S: Phosphorylation of transglutaminase 2 (TG2) at serine-216 plays a role in TG2 mediated activation of nuclear factor-kappa $B$ and in the downregulation of PTEN. BMC Cancer 2012, 12:277.

15. Kalluri R: EMT: when epithelial cells decide to become mesenchymal-like cells. J Clin Invest 2009, 119:1417-1419.

16. Thiery JP, Acloque $H$, Huang RY, Nieto MA: Epithelial-mesenchymal transitions in development and disease. Cell 2009, 139:871-887.

17. Sarrio D, Rodriquez-Pinilla SM, Hardisson D, Cano A, Moreno-Bueno G, Palacios J: Epithelial-mesenchymal transition in breast cancer relates to the basal-like phenotype. Cancer Res 2008, 68:989-997.

18. Geradts J, de Herreros AG, Su Z, Burchette J, Broadwater G, Bachelder RE: Nuclear Snail1 and nuclear ZEB1 protein expression in invasive and intraductal human breast carcinomas. Hum Pathol 2011, 42:1125-1131.

19. Kumar A, Xu J, Brady S, Gao H, Yu D, Reuben J, Mehta K: Tissue transglutaminase promotes drug resistance and invasion by inducing mesenchymal transition in mammary epithelial cells. PLoS One 2010, 5:e13390.

20. Lacerda L, Pusztai L, Woodward WA: The role of tumor initiating cells in drug resistance of breast cancer: implications for future therapeutic 
approaches. Drug Resist Updat 2010, 13:99-108.

21. Calcagno AM, Salcido CD, Gillet JP, Wu CP, Fostel JM, Mumau MD, Gottesman MM, Varticovski L, Ambudkar SV: Prolonged drug selection of breast cancer cells and enrichment of cancer stem cell characteristics. J Natl Cancer Inst 2010, 102:1637-1652.

22. Li X, Lewis MT, Huang J, Gutierrez C, Osborne CK, Wu MF, Hilsenbeck SG, Pavlick A, Zhang X, Chamness GC, Wong H, Rosen J, Chang JC: Intrinsic resistance of tumorigenic breast cancer cells to chemotherapy. J Nat Cancer Inst 2008, 100:672-679.

23. Mani SA, Guo W, Liao MJ, Eaton EN, Ayyanan A, Zhou AY, Brooks M, Reinhard F, Zhang CC, Shipitsin M, Campbell LL, Polyak K, Brisken C, Yang J, Weinberg RA: The epithelial-mesenchymal transition generates cells with properties of stem cells. Cell 2008, 133:704-715.

24. Chang JC, Wooten EC, Tsimelzon A, Hilsenbeck SG, Gutierrez MC, Tham YL, Kalidas M, Elledge R, Mohsin S, Osborne CK, Chamness GC, Allred DC, Lewis MT, Wong H, O'Connell P: Patterns of resistance and incomplete response to docetaxel by gene expression profiling in breast cancer patients. J Clin Oncol 2005, 23:1169-1177.

25. Kumar A, Gao H, Xu J, Reuben J, Yu D, Mehta K: Evidence that aberrant expression of tissue transglutaminase promotes stem cell characteristics in mammary epithelial cells. PLoS One 2011, 6:e20701.

26. Kumar A, Xu J, Sung B, Kumar S, Yu D, Aggarwal BB, Mehta K: Evidence that GTP-binding domain but not catalytic domain of transglutaminase 2 is essential for epithelial-to-mesenchymal transition in mammary epithelial cells. Breast Cancer Res 2012, 14:R4.

27. Cao L, M Shao, Schilder J, Guise T, Mohammad KS, Matei D: Tissue transglutaminase links TGF-beta, epithelial to mesenchymal transition and a stem cell phenotype in ovarian cancer. Oncogene 2012, 31:2521-2534.

28. Shostak K, Chariot A: NF-KB, stem cells and breast cancer: the links get stronger. Breast Cancer Res 2011, 13:214

29. Ai L, Skehan RR, Saydi J, Lin T, Brown KD: Ataxia-Telangiectasia, Mutated (ATM)/Nuclear Factor $\mathrm{k}$ light chain enhancer of activated B cells (NFKB) signaling controls basal and DNA damage-induced transglutaminase 2 expression. J Biol Chem 2012, 287:18330-18341.

30. Ghanta KS, Pakala SB, Reddy SD, Li DQ, Nair SS, Kumar R: MTA1 coregulation of transglutaminase 2 expression and function during inflammatory response. J Biol Chem 2011, 286:7132-7138.

31. Kumar S, Mehta K: Tissue transglutaminase constitutively activates HIF-1a promoter and nuclear factor-kB via a non-canonical path. PLoS One 2012, 11:e49321.

32. Park KS, Kim DS, Jeong KC, Kim SY: Increase in transglutaminase 2 expression is associated with NF-kappaB activation in breast cancer tissues. Front Biosci 2009, 14:1945-1951.

33. Kim DS, Park SS, Nam BH, Kim IH, Kim SY: Reversal of drug resistance in breast cancer cells by transglutaminase 2 inhibition and nuclear factorkappaB inactivation. Cancer Res 2006, 66:10936-10943.

34. Kim SY: Transglutaminase 2: a new paradigm for NF-kappaB involvement in disease. Adv Enzymol Relat Areas Mol Biol 2011, 78:161-195.

35. Kim Y, Eom S, Kim K, Lee YS, Choe J, Hahn JH, Lee H, Kim YM, Ha KS, Ro JY, Jeoung D: Transglutaminase II interacts with rac1, regulates production of reactive oxygen species, expression of snail, secretion of Th2 cytokines and mediates in vitro and in vivo allergic inflammation. Mol Immunol 2010 47:1010-1022.
36. Wilson WR, Hay MP: Targeting hypoxia in cancer therapy. Nat Rev Cancer 2011, 11:393-410.

37. Kim DS, Choi YB, Han BG, Park SY, Jeon Y, Kim DH, Ahn ER, Shin JE, Lee BI, Lee H, Hong KM, Kim SY: Cancer cells promote survival through depletion of the von Hippel-Lindau tumor suppressor by protein crosslinking. Oncogene 2011, 30:4780-4790.

38. Salgado R, Junius $S$, Benoy I, Van Dam P, Vermeulen P, Van Marck E, Huget $P$, Dirix LY: Circulating interleukin-6 predicts survival in patients with metastatic breast cancer. Int J Cancer 2003, 103:642-646.

39. Cieply B, Riley P 4th, Pifer PM, Widmeyer J, Addison JB, Ivanov AV, Denvir J, Frisch SM: Suppression of the epithelial-mesenchymal transition by Grainyhead-like-2. Cancer Res 2012, 72:2440-2453.

40. Antonyak MA, Miller AM, Jansen JM, Boehm JE, Balkman CE, Wakshlag JJ, Page RL, Cerione RA: Augmentation of tissue transglutaminase expression and activation by epidermal growth factor inhibit doxorubicin induced apoptosis in human breast cancer cells. J Biol Chem 2004, 279:41461-41467.

41. Antonyak MA, Li B, Regan AD, Feng Q, Dusaban SS, Cerione RA: Tissue transglutaminase is an essential participant in the epidermal growth factor-stimulated signaling pathway leading to cancer cell migration and invasion. J Biol Chem 2009, 284:17914-17925.

42. Wang Y, Ande SR, Mishra S: Overexpression of phospho mutant forms of transglutaminase 2 downregulates epidermal growth factor receptor. Biochem Biophys Res Commun 2012, 417:251-255.

43. Levental KR, Yu H, Kass L, Lakins JN, Egeblad M, Erler JT, Fong SF, Csiszar K, Giaccia A, Weninger W, Yamauchi M, Gasser DL, Weaver VM: Matrix crosslinking forces tumor progression by enhancing integrin signaling. Cell 2009, 139:891-906.

44. Hwang JY, Mangala LS, Fok JY, Lin YG, Merritt WM, Spannuth WA, Nick AM, Fiterman DJ, Vivas-Mejia PE, Deavers MT, Coleman RL, Lopez-Berestein G, Mehta K, Sood AK: Clinical and biological significance of tissue transglutaminase in ovarian carcinoma. Cancer Res 2008, 68:5849-5858.

45. Verma A, Guha S, Diagaradjane P, Kunnumakkara AB, Sanguino AM, LopezBerestein G, Sood AK, Aggarwal BB, Krishnan S, Gelovani JG, Mehta K: Therapeutic significance of elevated tissue transglutaminase expression in pancreatic cancer. Clin Cancer Res 2008, 14:2476-2483.

46. Badarau E, Collighan RJ, Griffin M: Recent advances in the development of tissue transglutaminase (TG2) inhibitors. Amino Acids 2013, 44:119-127.

47. Budillon A, Carbone C, Gennaro ED: Tissue transglutaminase: a new target to reverse cancer drug resistance. Amino Acids 2013, 44:63-72.

48. Pinkas DM, Strop P, Brunger AT, Khosla C: Transglutaminase 2 undergoes a large conformational change upon activation. PLOS Biol 2007, 5:e327.

49. Yuan L, Holmes TC, Watts RE, Khosla C, Broekelmann TJ, Mecham R, Zheng H, Izaguirre EW, Rich KM: Novel chemo-sensitizing agent, ERW1227B, impairs cellular motility and enhances cell death in glioblastomas. J Neurooncol 2011, 103:207-219.

doi:10.1186/bcr3371

Cite this article as: Agnihotri $\mathrm{N}$ et al: Tissue transglutaminase as a central mediator in inflammation-induced progression of breast cancer. Breast Cancer Research 2013, 15:202. 